\title{
Herniation through the broad ligament
}

\author{
Shahzeer Karmali MD, Robert K. Zurawin MD, Vadim Sherman MD
}

Previously published at www.cmaj.ca

A

healthy 35-year-old, multiparous woman presented to

the emergency department with nausea, vomiting and a painful, bloated abdomen. On examination, the patient was afebrile and passing flatus. She had a distended abdomen that was silent and tympanitic, with mild tenderness in the lower left quadrant. The results of basic laboratory investigations (i.e., complete blood count, electrolyte panel) were normal. Computed tomography showed dilated loops of distal small bowel in the lower abdomen consistent with a partial obstruction of the small bowel. A diagnostic laparoscopy showed an internal herniation of the small bowel through a $5-\mathrm{cm}$ defect in the broad ligament, between the round ligament and left adnexa (Figure 1A). After reduction of the herniated bowel, the broad ligament was cut to prevent recurrence.

First reported in an autopsy series in 1861 by Quain, ${ }^{1}$ herniation through a defect in the broad ligament remains an uncommon cause of intestinal obstruction, accounting for about $4 \%-7 \%$ of internal hernias. ${ }^{2}$ Cilley classified broadligament defects in three categories (Figure 1B). ${ }^{3}$ Our patient had a complete fenestration through the broad ligament, or type 1 defect, which is the most common type.

Defects of the broad ligament may be either acquired or congenital. Trauma resulting from pregnancy or delivery, pelvic inflammatory disease or surgery is an acquired cause. In nulliparous patients, such defects may result from spontaneous rupture of cystic structures within the broad ligament that are thought to be congenital remnants of the mesonephric or mullerian ducts. ${ }^{3}$ Herniation or obstruction of the small bowel is the most commonly reported complication. Ureteric herniation with obstruction $^{4}$ and ovarian torsion with gangrenous strangulation within defects of the broad ligament have also been reported. ${ }^{5}$

From the Division of General Surgery (Karmali), Department of Surgery University of Alberta, Edmonton, Alta.; and the Divisions of General Surgery (Sherman) and Obstetrics and Gynecology (Zurawin), Micheal E. DeBakey Department of Surgery, Baylor College of Medicine, Houston, USA

CMAJ 2009. DOI:10.1503/cmaj.090667
The management of internal hernia through the broad ligament is two-fold. First, using the Trendelenburg position, the incarcerated contents are gently reduced, and, if necessary, nonviable bowel is resected. Second, to prevent recurrent smallbowel obstruction, the defect can be closed (i.e., using a clip or suture $)^{6}$ or the broad ligament can be completely divided. ${ }^{7}$

The possibility of internal herniation through a defect in the broad ligament should be considered in women presenting with obstruction of the small bowel when more common causes (i.e., adhesions, neoplasms, groin hernias) have been excluded.

This article has been peer reviewed.

Competing interests: None declared.

\section{REFERENCES}

1. Baron A. Defect in the broad ligament and its association with intestinal strangulation. Br J Surg 1948;36:91-4.

2. Chapman VM, Rhea JT, Novelline RA. Internal hernia through a defect in the broad ligament: a rare cause of intestinal obstruction. Emerg Radiol 2003;10:94-5.

3. Cilley R, Poterack K, Lemmer J, et al. Defects of the broad ligament of the uterus. Am J Gastroenterol 1986;81:389-91.

4. Nackley AC, Yeko TR. Ureteral displacement associated with pelvic peritoneal defects and endometriosis. J Am Assoc Gynecol Laparosc 2000;7:131-3.

5. Bates GJ, Bennett IC, Furnival CM. A strangulated hernia through the broad ligament causing ureteric obstruction. J R Coll Surg Edinb 1983;28:335.

6. Varela GG, López-Loredo A, Garcia León JF. Broad ligament hernia-associated bowel obstruction JSLS 2007;11:127-30.

7. Takayama S, Hirokawa T, Sakamoto M, et al. Laparoscopic management of small bowel incarceration caused by a broad ligament defect: report of a case. Surg Today 2007;37:437-9. 